\title{
Global tuberculosis partnership says industrialized world must do more
}

Published at www.cmaj.ca on Oct.15

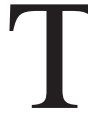

he World Health Organization (WHO) has unveiled a new blueprint to halve the $1.8 \mathrm{mil}$ lion deaths caused by tuberculosis (TB) each year but says it needs an additional US $\$ 14$ billion from industrialized world over the next five years to achieve that target.

The Global Plan to Stop TB 2011 2015: Transforming the Fight Towards Elimination of Tuberculosis, announced simultaneously at the World Health Summit in Berlin, Germany, and in Johannesburg, South Africa, proposes to increase tuberculosis spending to US\$47 billion from the US\$36 billion that the Stop TB Partnership had originally projected it would need over that five-year period to limit TB mortality rates. WHO estimates that nine million new cases of TB are still being diagnosed each year.

The US\$11-billion increase is largely a function of the partnership's plan to persuade the industrialized world to discontinue their "deceleration" of TB research and development, so as to develop new diagnostics, drugs and vaccines. The plan proposes to spend US\$9.8 billion over the next five years to entice more researchers back into the field (www.stoptb.org/assets /documents/global/plan/TB_GlobalPlan ToStopTB2011-2015.pdf).

It also proposes to increase funding for TB treatment to US\$36 billion over the five-year period, including US\$22.6 billion to treat new TB patients, US\$7.1 billion to treat patients with drug-resistant TB, US\$2.8 billion to treat HIV patients with TB, \$US4 billion to strengthen laboratory capacity within the 22 "high-burden" countries that account for $80 \%$ of the world's TB cases, and US\$400 million on administration.

United Nations Special Envoy to Stop Tuberculosis and former president

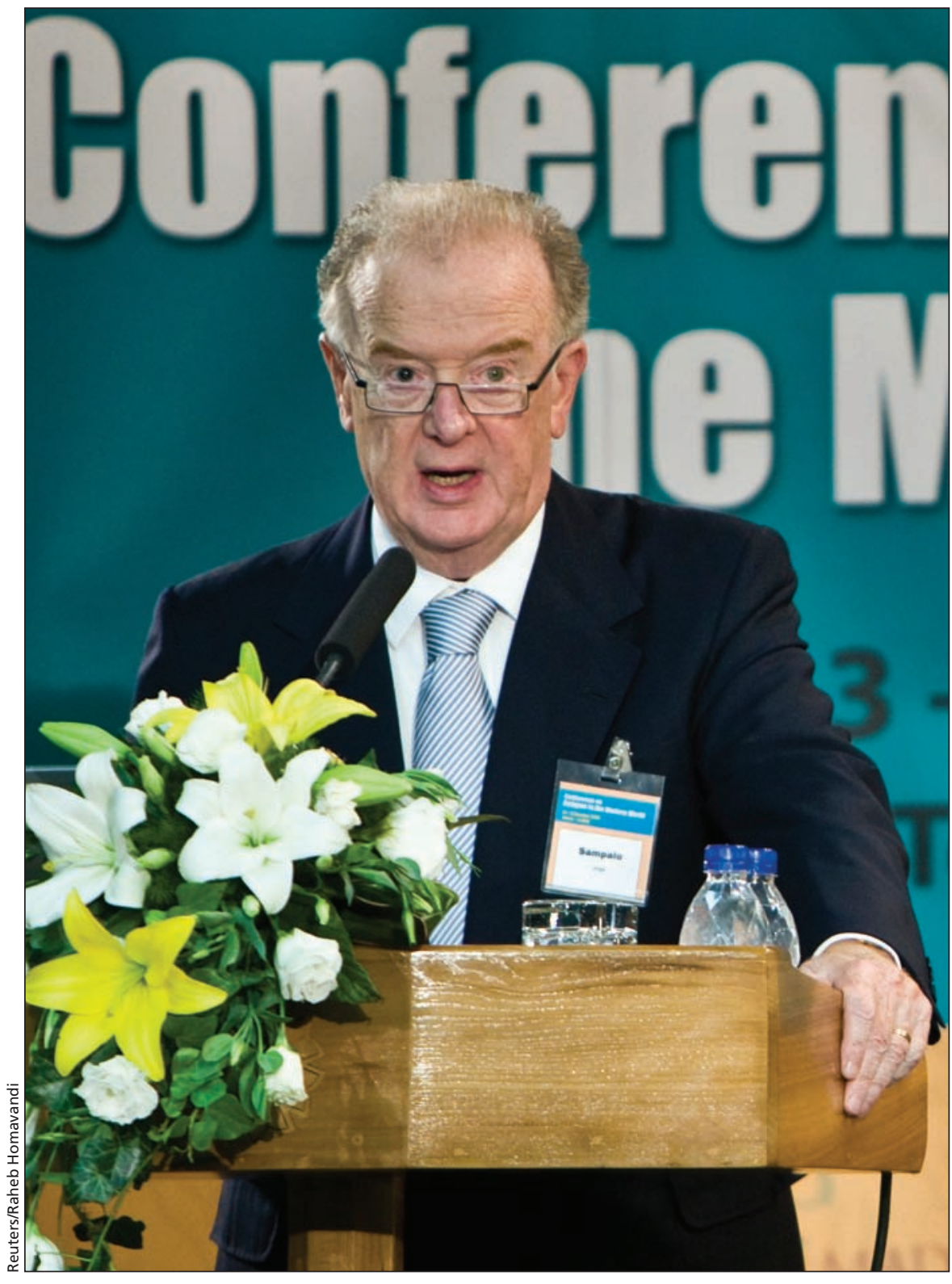

Emerging economic powers, particularly those with a high incidence of tuberculosis, and "rich oil producers" have to make greater contributions to the global tuberculosis effort, says United Nations Special Envoy to Stop Tuberculosis Jorge Sampaio.

of Portugal Jorge Sampaio told the summit that after taking into account current TB spending commitments, "there will be a gap of US\$14 billion for implementation, and some US\$10 billion for research and development."
Sampaio argued that emerging economic powers, particularly those with a high incidence of TB, and "rich oil producers" had to make a greater contribution to the global TB effort and thus, "strengthen solidarity between 
peoples and communities sharing the same culture and values."

"We need to bring on board new partners, create coalitions of interest among emerging and old powers to fight against tuberculosis as a matter of global security and sustainable development, not to mention as a fundamental issue of human rights," he said. "The world is undergoing great changes and a new world order is emerging. But we have not yet adopted our partnerships strategy to it and a shared agenda is still to be shaped."

"Therefore, it is time for action," Sampaio added. "If this new world order is to reflect the ongoing shift in the balance of economic power, it is time to call upon the new players to perform an increased role in addressing major issues and challenges, to revamp existing partnerships, to set a new shared agenda for co-operation."

Sampaio later told a press conference that the annual shortfall in funding for the new blueprint is US\$4.2 billion per year (US\$2.8 billion per year for TB treatment and US\$1.4 billion per year for research), which translates into a gap of US\$21 billion over five years.

But Dr. Hans Kluge, the WHO's director of health systems and public health, explains that the four so-called BRIC countries (Brazil, Russia, India and China), all of whom are among the 22 high-burden nations, are "expected" to increase their domestic spending on TB treatment to meet the obligation of endemic countries to provide at least half of the required funds domestically, as well as make a greater contribution to research and development.

Russia, for example, has already indicated that it will boost its spending by US $\$ 1$ billion and WHO is in negotiation with the remaining BRIC nations to increase their spending to a level commensurate with how endemic TB is within their borders, Kluge says. "That leaves $\$ 14$ billion for the high-income countries."

The standard treatment for TB, known as DOTS (Directly Observed Treatment, Short-Course) involves the use of isoniazic, rifampicin, pyrazinamide and ethambutol for two months, and then isonizid and rifampicin for a further four months. But because many people who contract TB do not follow the regimen, strains of multi-drug resistant (MDR) TB and extensively drugresistant TB have emerged. They require more expensive forms of treatment.

Sampaio said there are 600 new cases of multi-drug resistant TB per year, "mostly in South Africa. ... WHO estimates that in 2009, nearly half a million MDR cases emerged and 150000 deaths were caused by MDRTB. In some settings, over a quarter of all new TB patients are now being diagnosed with MDR-TB."

He also argued that "a quantum leap in TB research is critical to make a difference precisely in the lives of the poorest and most marginalized."

"The new global plan for the very first time sets a true TB research agenda," Sampaio said, adding that "fundamental science may seem disconnected from the urgent needs of the world's poor who are affected by disease. However, this is strategic short sightedness." - Wayne Kondro, CMAJ

DOI:10.1503/cmaj.109-3702 